\title{
Functional spermatid-like cells derived from the ground-state embryonic stem cells in vitro
}

\author{
Yan Zhang \& Yixun Liu* \\ State Key Laboratory of Stem Cell and Reproductive Biology, Institute of Zoology, Chinese Academy of Sciences, Beijing 100101, China
}

Received March 10, 2016; accepted March 16, 2016; published online March 25, 2016

Citation: Zhang, Y., and Liu, Y. (2016). Functional spermatid-like cells derived from the ground-state embryonic stem cells in vitro. Sci China Life Sci 59, 436-437. doi: 10.1007/s11427-016-5048-Z

Embryonic stem cells (ESCs) are pluripotent and can self-assemble to form cell clusters or embryoid bodies (EBs), which can then differentiate into all cell types of the three germ layers, as among which one is the primordial germ cell (PGCs) (Daley, 2007). In vivo PGCs are the gamete founder cells (the ooctyes and sperms), which transmit genetic information from one generation to the next generation to maintain mammalian life cycles. About $15 \%$ of couples in China are infertile; some of these infertility cases are caused by gametogenesis problems. It is therefore a major goal to produce functional gametes in culture for reproductive biology. However, the recapitulation of meiosis for male germ cells in culture has been hampered by a lack of appropriate techniques in vitro. By co-culturing ESCderived PGC-like cells (PGCLCs) with neonatal testicular somatic cells and then sequentially exposed these PGCLCs to morphogens and sex hormones, prof. Qi Zhou at Chinese Academy of Sciences and others obtained functional haploid cells in vitro (Zhou et al., 2016). This is the first time that scientists obtained ESC-derived spermatid-like cells (SLCs) in vitro, which undergo the key processes of meiosis, including methylation erasure, chromosomal synapsis and recombination, and finally differentiate into haploid cells.

The ground-state ESCs cultured in $2 \mathrm{i}$ medium (Ying et al., 2008) are required for the efficient derivation of PGCLCs. It has been reported that induced PGCLCs from ESCs can give rise to healthy sperms when transplanted into

*Corresponding author (email: liuyx@ioz.ac.cn) neonatal $K I T^{\mathrm{W}} / K I T^{\mathrm{W}-\mathrm{V}}$ mice lacking endogenous spermatogenesis (Hayashi et al., 2011). Zhou, Sha and their collaborator's work (Zhou et al., 2016) extended this finding and showed that PGCLCs from ground-state ESCs can complete meiosis in vitro when co-cultured with neonatal $K I T^{\mathrm{W}} / K I T^{\mathrm{W}-\mathrm{V}}$ testicular cells. The authors first induced epiblast-like cells (EpiLCs) from the ground-state ESCs, followed by inducing PGCLCs from EpiLCs, according to a protocol published previously (Hayashi et al., 2011). They showed that dynamics of histone modification occurs during PGCLCs induction in vitro. Analysis of imprinted regions revealed that methylation levels of both H19 and Snrpn in PGCLCs were lower than those in ESCs. This result indicates the erasure of imprinting in PGCLCs. Enriched PGCLCs were then cultured with neonatal $K I T^{\mathrm{W}} / K I T^{\mathrm{W}-\mathrm{V}}$ testicular cells. Early postnatal testicular cells of $K I T^{\mathrm{W}} / K I T^{\mathrm{W}-\mathrm{v}}$ provide a permissive environment for the initiation of meiosis by expressing low levels of CYP26B, which inhibits the entry of male germ cells into meiosis. After 3 days of co-culture in the presence of three morphogens (activin, BMPs, and RA), PGCLCs entered into meiosis with upregulation of Stra8 and downregulation of Blimpl and Stella. This reality was demonstrated by lineage tracing systems. The expression of these unique gene signatures resemble the behavior of PGCs in the E13.5 female genital ridge, which enter into meiosis in the following few days. More interestingly, after six days of co-culture supplemented with three morphogens, testis somatic cells migrated actively toward differentiating PGCLCs. Taken together, the co-culture conditions establish a microenviron- 
ment suitable for the initiation of meiosis in germ cells.

During spermatogenesis in mouse, sex hormones, including follicle-stimulating hormone (FSH) and testosterone (T), regulate the progress of meiosis through testis somatic cells (Hunter et al., 2012). After seven days of co-culture, 14\%-20\% spermatid-like cells (SPLCs) were derived from co-cultures supplemented with FSH, bovine pituitary extract and $\mathrm{T}$. This occurred in the absence of the above-mentioned three morphogens. In contrast, haploid cells were undetectable in the presence of FSH and T or FSH and BPE. The results indicate that three hormones are required for the initiation of meiosis.

Chromosome synapsis and recombination in meiosis play a key role in gamete development in mice. Both of these operations require the generation and resolution of DNA double-strand breaks (DSBs). By using PGCLCs-KIT $T^{\mathrm{W}}$ / $K I T^{\mathrm{W}-\mathrm{V}}$ testis cell co-cultures for analyzing meiotic progress, $90 \%$ of the primary spermatocytes entered the leptotene or zygotene stage of meiosis on day 8. These differentiating PGCLCs were positive for SPO11 and RAD51, thus indicating the generation of DSBs and the resolution of DSBs by homologous recombination. Moreover, on day 8 , broad $\gamma$-H2AX distribution throughout the nucleus in cells reflected an association with DSBs, which recapitulated meiosis in vivo. However, on day $10, \gamma-\mathrm{H} 2 \mathrm{AX}$ in $64 \%$ cells disappeared from the autosomes and accumulated on the unsynapsed sex chromosomes. This result indicates that cells on day 10 complete synapsis and enter the pachytene stage. This occurrence is similar to the corresponding spermatocytes in mice. On day $12,50 \%$ of the meiotic cells were on the diplotene stage. These results indicated that meiosis is synchronized in the majority of germ cells when undergoing synapsis and recombination.

Global gene expression profile clustering analyses showed that the similarity of in vitro-derived haploid cells to in vivo-derived ones and the birth rate of ICSI (intracytoplasmic sperm injection) with SLCs were lower than those of comparable spermatids in vivo. These results may be attributed to different transcription between induced haploid cells in vitro and round spermatids in vivo. Moreover, methylations of H19 and Snrpn in in-vitro-derived haploid cells were different from those of spermatids in vivo but were comparable with them. Compared with the genome-wide methylation data of sperms and oocytes (Shen et al., 2014), the proportions of high methylation sites (80\%) of tail tip cells from in-vitro-haploid cell-derived offsprings and normal mouse were both lower than that of sperms but higher than that of the oocytes. This result consists with the lower methylation levels in somatic cells. Furthermore, analyses of imprinted regions in SLCs-derived offsprings and control mouse revealed that all of the regions in both of them showed $50 \%$ methylation levels. These data showed that spermatids derived from the ground-state ESCs in vitro are functional.

Although basic features of the meiosis in differentiating PGCLCs and DNA methylation in SLCs are similar to those of germ cells at comparable stages in mouse, in-vitroderived germ cells show some features very different from male mouse germ cells. For example, the authors showed that a single PGCLC can form four SLCs. This implies that PGCLCs could directly enter into meiosis, a scenario which is similar to female PGCs at E13.5 but different from in vivo spermatogenesis through mitosis and meiosis. Other results also indicate that the behavior of differentiating PGCLCs resembles that of PGCs in the E13.5 female genital ridges. These interesting phenomena require further analysis. This current work represents another major contribution to in-vitro-derived germ cells through collaboration between Professor Qi Zhou and Professor Jiahao Sha. Mechanistic understanding of meiosis of the mammalian germline and the final goal of treating infertility will be improved by new and transformative methods in the future.

Compliance and ethics The author(s) declare that they have no conflict of interest.

Daley, G.Q., (2007). Gametes from embryonic stem cells: a cup half empty or half full? Science 316, 409-410

Hayashi, K., Ohta, H., Kurimoto, K., Aramaki, S., and Saitou M. (2011). Reconstitution of the mouse germ cell specification pathway in culture by pluripotent stem cells. Cell 146, 519-532

Hunter, D., Anand-Ivell, R., Danner, S., and Ivell, R. (2012). Models of in vitro spermatogenesis. Spermatogenesis 2, 32-43

Shen, L., Inoue, A., He, J., Liu, Y., Lu, F., and Zhang, Y. (2014). Tet3 and DNA replication mediate demethylation of both the maternal and paternal genomes in mouse zygotes. Cell Stem Cell 15, 459-470

Ying, Q.L., Wray, J., Nichols, J., Batlle-Morera, L., Doble, B., Woodgett, J., Cohen, P., and Smith, A. (2008). The ground state of embryonic stem cell self-renewal. Nature 453, 519-523

Zhou, Q., Wang, M., Yuan, Y., Wang, X., Fu, R., Wan, H., Xie, M., Liu, M., Guo, X., Zheng, Y., Feng, G., Shi, Q., Zhao, X.Y., Sha, J., and Zhou, Q. (2016). Complete meiosis from embryonic stem cell-derived germ cells in vitro. Cell Stem Cell 18, 330-340

Open Access This article is distributed under the terms of the Creative Commons Attribution License which permits any use, distribution, and reproduction in any medium, provided the original author(s) and source are credited. 